\section{EL MODELO DE TRIPLE HÉLICE DE RELACIONES UNIVERSIDAD, INDUSTRIA Y GOBIERNO: UN ANÁLISIS CRÍTICO}

\author{
Teresa González de la Fe \\ IUCPS, ULL \\ tgdelafe@ull.es
}

\section{TRIPLE HELIX MODEL OF RELATIONS AMONG UNIVERSITY, INDUSTRY AND GOVERNMENT: A CRITICAL ANALYSIS}

\begin{abstract}
In this essay, I attend to the Triple Helix Model of relations among university, industry and government, broadly used in innovation studies. The model was proposed by Henry Etzkowitz and Löet Leyersdorf and it has been very succesful as a heuristic for analysis of innovation process and as a normative guide for innovation policies. Moreover, its theoretical foundations are suited with perspectives from evolutive and institutionalist theories of economic science, at the same time that it takes in account sociological variables usually unattended in economic theory. By other hand, the accumulative knowledge about diverse experiences of triple helix more than one decade ago has shown that the Triple Helix model is adequated as a impeller for innovations not only directed to market but also to various social problems. In spite of it, the model has originated critics pointing to its supporting functions and its weaknesses for adecuated descriptions and explanations of complex processes of innovation.
\end{abstract}

KEY WORDS: Innovation, triple helix, university, industry, government relationships, sociology of innovation.

\section{Introducción. BREVE historia del MOdelo dE TRIPLE HÉLICE}

El enfoque de la Triple Hélice de relaciones UniversidadIndustria-Gobierno (UIG en adelante) puede verse como un complemento sociológico de los modelos económicos, y muchas veces "economisticos" (Albert y Laberge, 2007), en los estudios de la innovación. Dentro del marco general de la economía evolucionista y de las políticas liberales en los países más ricos, el desarrollo de la literatura procedente de la Triple Hélice forma parte de la evolución de los modelos (y de las políticas que se inspiran en ellos) sobre la ciencia, la tecnología y la industria. El interés se fue desplazando desde la I+D en las décadas de la posguerra de mediados del siglo XX, hasta el actual énfasis en la innovación como motor generador de riqueza en el XXI. La
RESUMEN: Se revisará el modelo de Triple Hélice de relaciones entre universidad, industria y gobierno, ampliamente usado en los estudios de la innovación. Impulsado por Etzkowitz y Leyersdorf, el modelo ha resultado fructífero como heuristica para el análisis de los procesos de innovación y como esquema normativo para políticas de innovación. Además, los fundamentos teóricos del modelo casan bien con los enfoques de las teorias evolucionista e institucionalista en economía, al tiempo que toman en cuenta variables sociológicas tradicionalmente descuidadas por la teoría económica. Por otro lado, la acumulación de conocimiento en torno a experiencias de triples hélices durante más de una década ha mostrado que el modelo sirve como impulsor de innovaciones no sólo destinadas al mercado sino también a diferentes problemas sociales. Pese a ello, ha suscitado críticas que señalan sus funciones legitimadoras y sus debilidades para la descripción y la explicación de los complejos procesos de las innovaciones.

PALABRAS CLAVE: Innovación, modelo de triple hélice, relaciones universidad, industria, gobierno, sociología de la innovación.

importancia de la innovación tecnológica es reconocida en la teoría económica desde Marx en adelante, especialmente en la década de 1950 (Leydesdorff, 2006b). Pero desde finales de los ochenta del siglo XX, ha sido creciente la presencia de la innovación en los discursos políticos sobre ciencia y tecnología que antes sólo hablaban de I+D. A la I+D se añadió la i de innovación, y los fondos para ciencia y tecnología, antes separados entre los ministerios de educación (universidad y centros públicos de investigación) e industria (empresas y sector privado), comenzaron a repartirse tomando en cuenta los resultados (especialmente en términos de rendimientos comerciales) de las investigaciones realizadas con fondos públicos. Lamo de Espinosa (2001) captó bien este cambio en los discursos políticos al señalar que la derecha se hizo innovadora al tiempo que la izquierda se hacia conservacionista. 
El origen remoto de estos cambios está en la crisis del petróleo de los años setenta y la recesión de los ochenta del pasado siglo, que tuvo entre otras consecuencias la de replantear el papel de la ciencia y la tecnología como solución para la dependencia energética y el desarrollo económico (Shinn, 2002). Este conjunto de modelos de desarrollo económico basado en la innovación tiene como telón de fondo la obra de Schumpeter $(1954,1942)$ y su concepción de la empresa como el locus de la innovación tecnológica, así como su uso del concepto de destrucción creativa para describir los cambios producidos por estas innovaciones. El punto de partida común de los nuevos modelos de innovación es la crítica al llamado "modelo lineal de innovación" que separa investigación fundamental e investigación aplicada, así como el énfasis en el acercamiento y la colaboración entre la investigación académica y la industria. El modelo, ya clásico pero aún influyente, de sistema nacional de innovación (Lundvall, 1992, 1988) dio pie a una amplia literatura académica con importantes repercusiones en las políticas emprendidas por organismos internacionales como la OCDE, la UNCTAD o la UE.

La extensa literatura académica sobre innovación que sigue a los trabajos de Lundvall, Freeman y Edquist, propone modelos de sistemas de innovación a diferentes niveles: sectorial (Malerba, 2002), tecnológico (Carlsson, 1997; Carlsson y Stankiewicz, 1991), regional (Braczyk et al., 1998; Cooke et al., 1997) o local; los cuales han servido de inspiración a numerosas de políticas de innovación. Por su parte, los organismos de gestión de esas políticas, tanto en el sector público como en el privado, han producido su propia literatura sobre el tema a través de informes y publicaciones de aportaciones a jornadas y congresos, la cual, sin ser del todo académica ni seguir los procedimientos propios de las publicaciones científicas, constituye un importante cuerpo teórico, y muchas veces doctrinal, sobre los procesos de innovación y sobre la planificación y el control de los cambios. Al mismo tiempo, tratan de operacionalizar las categorías de los modelos académicos para adaptarlos a las situaciones específicas de innovación en sus países, regiones o sectores (Jacobs, 2006).

El modelo de Triple Hélice ( $\mathrm{TH}$ en adelante) de relaciones UIG está en esta estela de producción académica destinada al uso de gobiernos y de organismos de financiación de la investigación. Su aportación distintiva es que procede de la sociología. Es un enfoque sociológico para el análisis de la innovación (Etzkowitz, 1994, Leydesdorff y Etzkowitz, 1996) y es, al mismo tiempo y sobre todo, una orientación para las políticas de innovación, una guía para lograr un desideratum. Se propone como complemento sociológico del enfoque de la economía evolutiva del cambio tecnológico tomando como elementos a la reflexividad y la comunicación (Leydesdorff, 2005). Es por ello un modelo prescriptivo para impulsar la innovación, al tiempo que es un análisis descriptivo de casos exitosos de innovación (Viale y Ghiglione, 1998). En este sentido, la TH es una propuesta más para ofrecer razones que justifiquen y legitimen decisiones políticas en materias de innovación y economía del conocimiento, al igual que otros enfoques como el de Sistemas de Innovación (Lundvall et al., 2002) o el Modo 2 de producción de conocimiento (Gibbons et al., 1994; Nowotny et al., 2001).

El modelo de TH se centra en el análisis de las relaciones e interacciones mutuas entre las universidades y los entornos científicos como primera pala de la hélice, las empresas e industrias como segunda pala y las administraciones o gobiernos como tercera pala. Atiende a las interacciones y comunicaciones entre actores e instituciones de las tres palas de la hélice, pues asume que la innovación surge de las interacciones mutuas entre ellas: el potencial para el conocimiento innovador, los recursos económicos y las posibilidades de mercado, y las normas e incentivos de las políticas públicas de innovación. La imagen de una triple hélice (inspirada en la biología) es una metáfora para expresar una alternativa dinámica al modelo de innovación imperante en las políticas de los años ochenta del siglo veinte, al tiempo que visualiza la complejidad inherente a los procesos de innovación (Etzkowitz, 2003).

En la $T H$, a diferencia de otros modelos, no hay un libro de referencia en el que se expongan sus fundamentos y conceptos (Shinn, 2002), pues sus teóricos principales, Loet Leydesdorff de la Universidad de Amsterdam y Henry Etzkowitz en el Science Policy Institute de la Universidad estatal de Nueva York en Purchase, han ido desarrollando el enfoque en varios trabajos conjuntos y en publicaciones por separado y con otros autores. Siendo consecuentes con sus ideas, ambos autores actúan como consultores y como staff académico de diversos organismos de distintos países para diseñar y emprender sus políticas de innovación. Pese a que ambos han realizado análisis comparativos del enfoque de la TH frente a otros similares (por 
ejemplo, Etzkowitz y Leydesdorff, 2000; Leydesdorff, 2000; Leydesdorff y Etzkowitz, 2001a; Leydesdorff, 2006b), sus principales trabajos han consistido en la exposición de su propio enfoque (Etzkowitz, 1994; Leydesdorff y Etzkowitz 1996; idem (eds.), 1997; Leydesdorff et al., 2006), en su aplicación a distintos contextos (Etzkowitz, 2002a; 2002b; 2003) y en hacerlo compatible con las corrientes básicas de los estudios de la innovación (Leydesdorff, 2001; 2005; 2006a).

Desde 1997 una red internacional de investigación sobre el modelo de la TH se reúne cada dos años en una Conferencia en la que se exponen sus aplicaciones y desarrollos. La primera se celebró en Amsterdam y las siguientes en Nueva York (1999), Río de Janeiro (2001), Copenhague (2003), Turín (2005) y Singapur (2007), estando la próxima convocada en Glasgow para 2009. La diversidad de continentes de las ciudades de acogida visualiza la amplitud global de la red en torno al modelo de TH. A estas citas bianuales hay que añadir diversos encuentros internacionales sectoriales que tienen lugar entre una y otra, lo que muestra el dinamismo de la red, así como su capacidad de atracción de investigadores y decisores políticos de países o áreas con situaciones económicas muy diferentes en lo que respecta a la innovación como los EE.UU. (Etzkowitz, 1997), la UE (Leydesdorff y Fritsch, 2006; Molas et al., 2007; Viale y Campodall'Orto, 2002) los paises emergentes de América Latina (Etzkowitz y Carvalho de Mello, 2004; Etzkowitz, Carvalho de Mello y Almeida, 2005) y Asia (Etzkowitz y Zhou, 2006; Irawati, 2006; Kadiman, 2006) o el Magreb (Saad y Zawdie, 2005).

El modelo TH tiene su origen en la universidad empresarial surgida en los Estados Unidos donde existe una amplia tradición de colaboraciones entre los entornos académicos e industriales, entre la universidad y las agencias gubernamentales y entre el gobierno y las industrias (Etskowitz, 1989). Las relaciones entre las esferas de la ciencia, el Estado y las empresas, sin embargo, se remontan al origen mismo de la ciencia moderna (Merton, 1970, citado por Etzkowitz, 2003), siendo Internet únicamente un producto reciente de estas colaboraciones surgidas del proyecto DARPA'. De hecho, la historia de la universidad moderna, la humboldtiana, que integra enseñanza e investigación como actividades públicas, es un antecesor de la TH. En los EE.UU. existe una larga tradición de universidades agrícolas, de las cuales el MIT es una continuación, y la Segunda Guerra Mundial propició un escenario de estrechas colaboraciones entre los gobiernos y las universidades en todos los paises involucrados en la contienda (Etzkowitz, 1989, 1994, 2003; Martin y Etzkowitz, 2000).

Sin embargo, sólo a finales del pasado siglo los paises más industrializados comenzaron a intensificar la base de conocimiento de sus economías, especialmente a partir del cambio de paradigma tecnológico hecho posible por el desarrollo de las nuevas tecnologías de la información y las comunicaciones (TIC). La innovación se convierte en el elemento que dota de ventajas competitivas a las empresas, y la investigación científica y tecnológica pasa a ser la base para la creación de riqueza y para el desarrollo económico. Surge el concepto de "economía basada en el conocimiento" (OCDE, 1996) que se convierte en el objetivo de la mayor parte de las políticas económicas de los paises posindustrializados, como sucede en la Unión Europea con los compromisos basados en la cumbre de Lisboa del año 2000 (Leydesdorff, 2006a).

Paralelamente, desde las ciencias sociales se desarrollan los análisis y modelos para explicar y dar cuenta de la innovación como elemento principal de este nuevo tipo de economía, hecha posible por las TIC y el proceso de mundialización de los mercados y las relaciones conocido como globalización. Como Leyersdorff (2005) señala, "las distintas metáforas en el estudio de los sistemas de innovación basados en el conocimiento pueden ser consideradas como apreciaciones teóricas de una dinámica compleja desde diferentes perspectivas y con objetivos potencialmente diferentes". Debido a ello, cada modelo de estudio de la innovación construye el sistema de innovación al que se dirige, tanto en lo relativo a las relaciones con su entorno como respecto a las interacciones entre sus subsistemas. De ahí que cada modelo analítico dependa de las definiciones establecidas y que los cambios en las definiciones conduzcan a innovaciones en las perspectivas. El modelo de la TH es la metáfora sociológica para el estudio de la innovación y se desarrolla en la estela de la sociología de la ciencia de Merton, la teoría sociológica de sistemas de Parsons y Luhmann y los estudios sociales de la ciencia y la tecnología, tratando de superar el sesgo hacia lo microcaracterístico de las sociologías del conocimiento científico (González y Sánchez, 1988).

ARBOR CLXXXV 738 julio-agosto [2009] 739-755 ISSN: 0210-1963

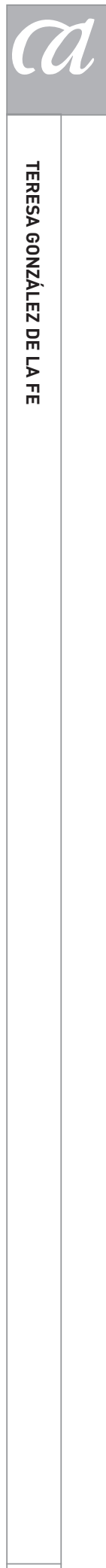

741 


\section{Marco teórico de la Triple Hélice}

En este apartado se tratará el armazón teórico de la TH que permitirá una mejor comprensión de sus logros y sus deficiencias. Aunque al definir los principales términos y conceptos usados por los autores del modelo, en cierto modo ya se están adelantando algunas de sus hipótesis y resultados, parece necesario explicitar los significados asignados a los conceptos más comunes que se repiten constantemente en los trabajos de sus dos autores más representativos.

Antes se expondrá una visión sintética del modelo de la TH a fin de encuadrar y dar sentido a sus conceptos e hipótesis. La TH es un modelo cuyo marco general es la economía evolutiva y los enfoques institucionalistas en teoría económica, complementado con una perspectiva sociológica sobre los procesos de innovación. En el caso de Etzkowitz, se puede rastrear el influjo de Mead y del interaccionismo pragmatista en su atención hacia las dimensiones micro, así como en el uso de un soporte analítico y de unas categorias de inspiración sistémica parsoniana (por ejemplo, Etzkowitz, 1989), que le sirven de puente entre las dimensiones macro y micro del estudio de los procesos de innovación. En el caso de Leydesdorff, es más fuerte la huella europea de la 4S -Society for Social Study of Science- y de los estudios posmodernos de la ciencia y la tecnología, así como un saludable interés por la medida y la operacionalización de las variables más importantes del modelo de TH (por ejemplo, Leydesdorff, 2003; Leydesdorff y Meyer, 2003 y 2006)².

Ambos autores comparten el enfoque aplicado y dirigido a la intervención pública en el marco de las políticas de ciencia e industria de la OCDE, la Unión Europea y los órganos federales y estatales de los EE.UU. y otros países. El modelo de TH se presenta como un esquema facilitador de la planificación pública de actuaciones, de la toma de decisiones y de la evaluación de la acción pública en materias tan importantes como la industria, la enseñanza superior y la universidad, y la investigación científica y tecnológica. De ahí que analice experiencias exitosas de innovaciones y que proponga espacios de intervención destinados al fomento de las innovaciones en la economía del conocimiento (Etzkowitz y Carvalho de Mello, 2004), así como las innovaciones de orden social y cultural propias de las sociedades del conocimiento (Etzkowitz, 2002b; Etzkowitz y Zhou, 2006).
Aunque sin asumir explícitamente los compromisos teleológicos de Parsons ${ }^{3}$, la concepción de los entornos de los sistemas de innovación es también deudora del marco analítico parsoniano de unas sociedades que evolucionan desde un esquema $\mathrm{AGIL}^{4}$ dependiente de la energía, hacia un esquema LIGA que pivota sobre los conocimientos, usando como mecanismos evolutivos la diferenciación funcional y la especialización institucional.

\subsection{Conceptos principales}

La innovación es el concepto central pues se trata de impulsar la innovación y de medir las innovaciones. La innovación se considera como un proceso inestable que por definición opera en la interfaz. Si bien una innovación es una novedad aislada, normalmente imprevista, lo importante es la organización y el control de la producción de innovaciones relevantes desde el punto de vista económico o social. Por ello, el interés no se centra en el fenómeno aislado de las innovaciones sino en los sistemas de innovación. Los sistemas de innovación se consideran como dinámicas de cambio tanto en los sistemas de producción como de distribución (Leydesdorff y Etzkowitz, 2001a) y tienen lugar en el seno de economías basadas en conocimiento. A su vez, el continuo aumento, tanto en el volumen como en la magnitud de su peso en el PIB de los estados, de productos que incorporan conocimiento conforma el tejido de la sociedad del conocimiento, concepto éste que también requiere de precisión y mejor definición (González 2002), dado su doble papel descriptivo y prescriptivo en los discursos académicos y políticos.

Desde la perspectiva neoevolucionista, los sistemas de innovación presentan problemas de diverso tipo debido, por una parte, a su naturaleza cambiante y emergente $y$, por otra, a que las innovaciones se producen en los intersticios o interfaces entre los diversos subsistemas de agentes implicados: universidad, industria y gobierno. Por ello, Leydesdorff (2001) señala que, al tratar de responder a la pregunta sobre la unidad de análisis más adecuada para el estudio de la tecnología y la innovación, los distintos enfoques usan metáforas diferentes según sus intereses teóricos y los objetivos de sus análisis, lo que genera la ilusión de un objeto estable que permite a los gestores legitimar las políticas de ciencia y tecnología. 
La TH adopta como punto de partida que "la innovación no es una unidad estable de análisis, sino una unidad de operación en un interfaz" (Leydesdorff, 2001: 2). Los sistemas de innovación se producen como resultado de las interacciones efectuadas en los intersticios de los tres subsistemas implicados. Ello permite la superación del marco de los sistemas nacionales de innovación como unidad relevante de análisis, y considerar los sistemas de innovación emergentes (caracterizados por resultados) en términos de una hiper red sobre las redes que lo constituyen (disciplinas, industrias y gobiernos nacionales) (Leydesdorff y Etzkowitz, 2001). La metáfora del barco de Neurath es citada a propósito (Leydesdorff y Etzkowitz, 2001a y b, Leydesdorff, 2006b): la economía del conocimiento transforma "el barco mientras la tormenta ruge en mar abierto" (Leydesdorff y Etzkowitz, 2001a: 20).

Para Leydesdorff el modelo de la TH ensancha el antiguo estructural-funcionalismo "introduciendo la noción de significado desde el interaccionismo simbólico: las funciones sociales están construidas discursivamente y pueden ser desconstruidas y reconstruidas reflexivamente" (2001a: 5). De este modo, las funciones están constantemente en reconstrucción y los elementos institucionales de los sistemas se generan mediante estas operaciones reflexivas. El espacio para las consecuencias no queridas ni previstas es amplio, pues en las economías basadas en el conocimiento predominan la reflexividad y la incertidumbre.

La interfaz en la que operan los sistemas de innovación está compuesta por la zona de encuentro entre los subsistemas de la universidad, de las industrias basadas en conocimiento y de los gobiernos, que constituyen las tres palas de la hélice, como se muestra en la figura siguiente.

Los motores de la innovación son las relaciones e interacciones a dos o tres bandas entre estos subsistemas de acción. Los mecanismos que operan son la asunción institucional de otros roles y la emergencia de organizaciones híbridas en un contexto cultural (de normas y valores) que propicie y fomente este tipo de procesos de cambio. Las organizaciones se conciben como generadoras de fines y de recursos, mientras que las instituciones proporcionan valores y normas. Mediante la asunción institucional de otros roles emergen cambios y resultados nuevos basados en conocimiento científico y tecnológico que traspasan las tradicionales funciones desempeñadas por estos ámbitos
Figura 1. Tomada de Etzkowitz y Leydesdorff (2000).

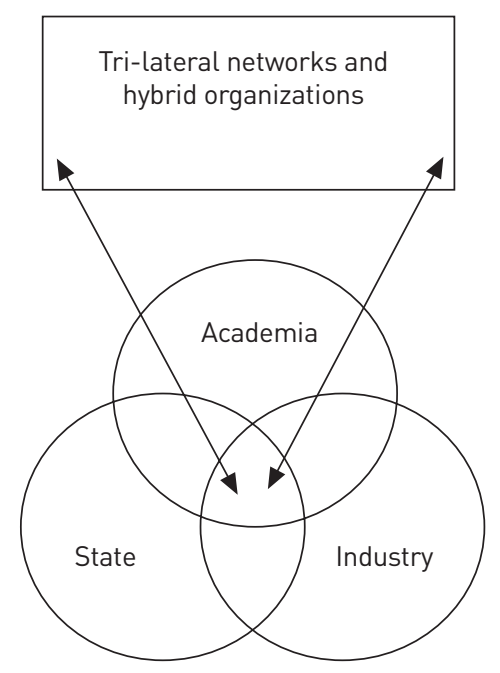

(industrias que hacen ciencia, investigadores académicos que forman empresas). Por su parte, las organizaciones híbridas son el resultado de estos cambios de roles al ser empresas académicas, organizaciones de interfaz 0 agencias de desarrollo gubernamentales.

El papel de las universidades es estratégico en todo el proceso, pues se requiere que las universidades desempeñen nuevas funciones y que se generen instituciones diferenciadas. La tercera función de la universidad en las economías y sociedades de conocimiento es contribuir al desarrollo económico y social local mediante innovaciones basadas en conocimientos. Esta función, dicen, se suma a las dos desempeñadas por la universidad humboldtiana de enseñanza e investigación. Etzkowitz y Leydesdorff (2000) anuncian una tercera revolución académica en marcha ya en muchas universidades. Éstas son las universidades emprendedoras que asumen la creación de empresas o de nidos empresariales en sus laboratorios e instalaciones, dando lugar a un nuevo tipo de personal universitario y a un tipo nuevo de investigador: el científico-empresario. Esta tercera revolución académica, un tanto retóricamente comparada con la próxima "gran transformación" (Viale y Etzkowitz, 2004), genera conocimiento "polivalente" como resultado de un centro de gravedad formado por implicaciones teóricas, prácticas e interdisciplinares. Este conocimiento "polivalente" se representa, con otra metáfora biológica, como el ADN de la triple hélice, resultado 
de las relaciones cambiantes entre conocimiento tácito y conocimiento codificado. Son disciplinas hibridas que han surgido como sintesis de intereses teóricos y prácticos, como la ciencia de la computación, la ciencia de los materiales o la nanotecnología (Leydesdorff y Etzkowitz, 2001a).

Los estudiantes se ven como inventores potenciales, que representan un flujo dinámico de "capital humano" en los grupos académicos de investigación frente al carácter estático de los laboratorios industriales y los institutos de investigación. Este flujo constante asegura la primacía de las universidades como fuentes de innovación. La combinación de continuidad y cambio, de memoria organizativa e investigadora con nuevas personas y nuevas ideas a través del paso de generaciones de estudiantes, proporciona a las universidades su ventaja comparativa especifica (Leydesdorff y Etzkowitz, 2001a).

La interpretación evolutiva del modelo TH supone que, dentro de contextos locales específicos, las universidades, el gobierno y la industria están aprendiendo a fomentar el crecimiento económico a través del desarrollo de "relaciones generativas" (Leyersdorff y Etzkowitz, 1997), es decir, relaciones recíprocas libremente vinculadas e iniciativas conjuntas que persisten a lo largo del tiempo y que dan lugar a cambios en el modo en que los agentes llegan a concebir su entorno y la manera de actuar dentro de él. El modelo de la TH no sólo se presenta como una alternativa más viable y coherente de las nuevas condiciones en que se desarrolla (se debe desarrollar) la ciencia y la tecnología (superadora de los modelos del Sistema Nacional de Innovación y del Modo 2 de producción de conocimiento), sino que considera, además, que las triples hélices (y el Modo 2) son ejemplos de lo que las universidades y el desarrollo de la ciencia y la tecnología han significado históricamente desde su aparición.

Leydesdorff y Etzkowitz (2001a) sostienen que el Modo 1 de producción de conocimiento -la ciencia académica basada en el modelo lineal de investigación básica o fundamental e investigación aplicada- es una ideología surgida en un momento histórico determinado en el que los totalitarismos de diverso signo amenazaban la libertad de investigación. El Modo 2 no es nuevo: es el formato original de la ciencia antes de su institucionalización académica en el siglo XIX (Leydesdorff y Etzkowitz, 2001a:
20). El Modo 1 es un constructo ideológico elaborado para justificar la autonomía para la ciencia, especialmente en los tiempos en que la ciencia era una institución frágil y necesitada de ayuda.

El núcleo de la tesis de la TH es la expansión del conocimiento en la sociedad y de la universidad en la economía (Etzkowitz, 2002b). Se postula un proceso de doble transformación de la universidad: expansión de sus misiones para incorporar el desarrollo económico y social y un cambio organizativo desde el individuo a la organización en cada misión. En este proceso, la TH se propone como una estrategia para aumentar el capital social y rellenar las brechas tecnológicas. Como ejemplos de estos procesos, se hace referencia al esquema de investigación en red en Canadá (Albert y Laberge, 2007) y la Unión Europea o el aumento de la incubación en Brasil (Etzkowitz y Carvalho de Mello, 2004; Etzkowitz, Carvalho de Mello y Almeida, 2005; Etzkowitz 2002a). En todos los casos, la TH actúa como modelo y motor de desarrollo.

La "capitalización del conocimiento", que sucede en paralelo con la "cognitivización del capital", se refiere a que se crean nuevas formas de capital se crean sobre la base de la interacción social ("a quién conoces") o de las actividades intelectuales ("qué sabes") y son intercambiables (Etzkowitz, 2003). De ahí que el capital financiero surja sobre el capital social e intelectual acumulado, y que éstos se redefinan en tanto que las universidades interactúan más intensamente con las industrias y los gobiernos.

Las incubadoras adquieren una relevancia cada vez mayor, dado que los nuevos productos y las nuevas empresas se basan cada vez más en conocimiento incubado. La transformación de los lazos universidad-industria es paralelo al hecho de que las empresas incubadas dejaran de ser casos aislados y pasaran a constituir redes. Este cambio viene apoyado por cambios en el entorno regulativo y en los programas de financiación, lo que favorece las interacciones UIG. (Etzkowitz, 2002a).

Por último, para la TH la legitimación clásica de la investigación científica basada en su contribución a la cultura permanece aún, y los objetivos militares y de salud siguen también como estímulos poderosos para financiar la investigación. Sin embargo, la legitimación futura de la investigación científica, que mantendrá la financiación de 
alto nivel, descansa en que supone una fuente creciente de nuevas líneas de desarrollo económico (Leydesdorff y Etzkowitz, 2001a). En este desiderátum se apoya la TH como "doctrina" o "ideología". Una vez vistas las ideas principales de la Th, se atenderá a las afirmaciones teóricas y los modelos de análisis más importantes.

\subsection{Teoría y modelos}

La tesis de la Triple Hélice postula que la interacción universidad-industria-gobierno es la clave para mejorar las condiciones para la innovación en una sociedad basada en el conocimiento. Esto supone que la universidad juegue un importante papel como fuente de nuevo conocimiento y nueva tecnología, dada su función de principio generador de las economías del conocimiento. La tesis es desarrollada en diez proposiciones (Etzkowitz, 2003: 296-299):

1. La fuente de la innovación descansa en las redes y acuerdos entre las tres esferas institucionales de la TH y no en alguna de ellas por separado.

2. La invención de innovaciones organizativas, de nuevos ordenamientos sociales y de nuevos canales para la interacción llega a ser tan importante para acelerar la innovación como la creación de dispositivos físicos. Ejemplos de estas innovaciones sociales son las incubadoras de empresas, los parques de la ciencia o las redes, las cuales Ilegan a ser fuentes de actividad económica, de formación de comunidad y de intercambio internacional.

3. El modelo interactivo de innovación es resultado de la interacción entre las dinámicas lineal y lineal inversa de transferencia de conocimiento. Cuando la tecnología se produce en la academia se da el paso del modelo lineal al lineal asistido (por ejemplo, con las empresas de las incubadoras). Cuando sucede en el modelo lineal inverso que comienza en las industrias o los problemas sociales, proporciona el punto de partida para nuevos programas de investigación y nuevas disciplinas.

4. La "capitalización del conocimiento" sucede en paralelo con la "cognitivización del capital", lo que significa que los procesos de creación de conocimiento y de creación de capital van en tándem, a medida que se inventan nuevas medidas de financiación de investigaciones arriesgadas y a medida que las empresas anidadas que transforman el conocimiento en capital dan origen a innovaciones organizativas.
5. La formación de capital sucede en dimensiones nuevas en la medida en que se crean diversas formas de capital que se transmutan en otras: financiero, social, cultural e intelectual. Las nuevas formas de capital se crean sobre la base de la interacción social ("a quién conoces") o las actividades intelectuales ("qué sabes") y son intercambiables. El capital financiero surge sobre el capital social e intelectual acumulado y éstos se redefinen en tanto que las universidades interactúan más intensamente con las industrias y el gobierno.

6. La globalización se descentraliza y tiene lugar a través de redes regionales entre universidades así como a través de corporaciones multinacionales y organizaciones internacionales. Las nuevas configuraciones se convierten en la base de un proceso continuo de formación de empresas, diversificación y colaboraciones entre competidores.

7. Los paises en desarrollo y las regiones tienen la posibilidad de hacer progresos rápidos basando sus estrategias de desarrollo en la construcción de nichos de fuentes de conocimiento apoyados por la economía local. Los ordenamientos políticos y sociales basados en principios de equidad y transparencia son la base para un rápido desarrollo en un entorno estable. Las universidades y las redes de incubadoras pueden usarse a la vez para adaptar tecnologías avanzadas para solucionar problemas locales y para avanzar las fronteras de la investigación en áreas especiales y transferir al exterior las innovaciones locales.

8. Las reorganizaciones entre esferas institucionales, sectores industriales y estados-nación son inducidas mediante oportunidades en nuevas tecnologias. Éstas emergen en un flujo constante desde síntesis entre innovaciones interdisciplinares previas.

9. Las universidades se convierten de forma creciente en la fuente de desarrollo económico regional y las instituciones académicas se reorientan o se crean con este fin.

10. Lo que distingue a una región Triple Hélice es la capacidad para hacer una transición de un paradigma tecnológico a otro cuando el anterior régimen se agota. Las interacciones Triple Hélice institucionalizadas y renovadas a través de generaciones de tecnologías son la base de redes aparentemente autoorganizadas de innovación.

ARBOR CLXXXV 738 julio-agosto [2009] 739-755 ISSN: 0210-1963

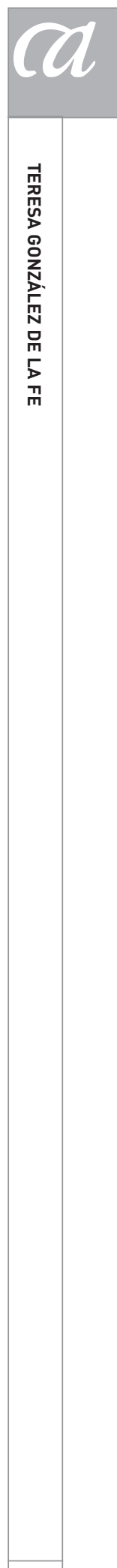

745 
Estas diez proposiciones son, al mismo tiempo, propuestas para emprender acciones destinadas a potenciar la innovación. Sin embargo, en la medida en que se realizaron hace más de un lustro, habrá que ver cómo se formularian o matizarian en momentos de recesión económica como los actuales. Por ejemplo, el punto 5 relativo a la formación del capital o el 6 referido a la descentralización de la globalización, también están necesitados de revisión en momentos de cierres masivos de empresas y aumento lineal del desempleo. Dado el carácter desiderativo de estas tesis, constituyen tanto un cuerpo de teoria para emprender medidas empíricas en diversas fases temporales, como un cuerpo doctrinal que proporciona un argumentario de buenas razones para emprender acciones políticas para la innovación.

Por otra parte, la TH proporciona un modelo de análisis para el nivel de la estructura social que permite explicar el Modo 2 como una estructura históricamente emergente para la producción de conocimiento científico y su relación con el Modo 1 (Leydesdorff y Etzkowitz, 2001a:25-7). Este modelo puede sintetizarse en seis puntos que recogen las principales relaciones entre las variables:

1) Los acuerdos entre industria y gobierno no necesitan ser conceptualizados exclusivamente como gobiernos nacionales y sectores industriales específicos: se producen toda clase de entrecruzamientos de alianzas estratégicas entre estos sectores actuando a diversos niveles y con diferentes organizaciones. Ejemplo: el Airbus.

2) La fuerza motriz de las interacciones ha de especificarse como la expectativa de beneficios. "Beneficio" puede significar cosas diferentes para los diversos actores implicados. Analíticamente, los motivos no han de ser conceptualizados como causas ex ante, sino en términos de expectativas que sólo pueden ser evaluadas ex post. "Desde la perspectiva evolucionista, la selección (ex post) está determinada por la estructura, mientras que la variación puede ser azarosa" (ibid., p. 25).

3) Al fundamentar el modelo en términos de expectativas se deja espacio para las incertidumbres y los procesos de oportunidad. Los portadores institucionales se reproducen en tanto que han sido funcionales hasta ahora, pero puede esperarse que las negociaciones conduzcan a experimentos que pueden ser institucionalizados posteriormente. Esto permite especificar un modelo de etapas de innovación que no tiene que corresponder con la teoría del ciclo de vida de un producto. Una innovación exitosa cambia la estructura de oportunidad para los actores institucionales implicados $y_{1}$ a su vez, se espera que los cambios estructurales cambien las dinámicas.

4) La expansión del sector de la educación superior y la investigación académica ha dotado a la sociedad de un mundo en el que diferentes representaciones pueden ser contempladas y recombinadas de forma sistemática. Por ejemplo, la "innovación de escritorio" (Kaghan y Barett, 1997, cit. Leydesdorff y Etzkowitz, 2001: 26) como diferente a la innovación de laboratorio. Las economías intensivas en conocimiento no pueden basarse en medidas simples de maximización de beneficios, sino que las funciones de utilidad han de encajar con las estructuras de oportunidad. A lo largo del tiempo, las estructuras de oportunidad son recursivamente conducidas por las contingencias de las tecnologías predominantes y posibles. A medida que la hélice opera, el factor capital humano se desarrolla más en curvas de aprendizaje y como antídoto al riesgo de desempleo tecnológico.

5) El modelo también explica por qué las tensiones no necesitan ser resueltas. Una resolución dificultaría las dinámicas de un sistema que vive de las perturbaciones e interacciones entre sus subsistemas. De este modo, se espera que los subsistemas se reproduzcan. Cuando se abre la "caja negra" se encuentra el Modo 1 dentro del Modo 2 y el Modo 2 dentro del Modo 1. El sistema no está ni del todo integrado ni del todo diferenciado, sino que actúa en los entrecruzamientos de las diferenciaciones fraccionales y las integraciones locales. Usando el modelo se puede empezar a entender por qué el régimen global se muestra en instancias progresivas, mientras que las instancias locales nos informan sobre desarrollos globales en términos de excepciones que son replicadas y construidas (ibid, p. 26).

6) La cuestión crucial de los medios de intercambio -expectativas económicas (en términos de beneficio y crecimiento), expectativas teóricas, evaluación de lo que puede realizarse dadas las restricciones geográficas e institucionales- es que han de ser relacionados 
y convertidos unos en otros. Las hélices se comunican recursivamente en el tiempo en términos del código propio de cada una, pues, reflexivamente, sólo pueden tomar el rol de cada otro hasta cierto punto.

Estos seis puntos sobre la estructura social de la innovación posibilitan la creación de modelos para el análisis de casos empíricos de innovación, al tiempo que para la construcción de indicadores que hagan posible la medición de variables tan importantes como las alianzas entre los sectores estratégicos, las expectativas de beneficios diferenciales, la influencia de incertidumbres, la emergencia de experimentos organizativos y su posible institucionalización en el marco de actuaciones públicas. etc.

Etzkowitz (2002) propone un modelo para la intervención en las políticas públicas de desarrollo regional basado en un esquema de tres etapas sucesivas: espacio de conocimiento, espacio de consenso y espacio de innovación, que él mismo resume en el cuadro siguiente.

\section{MARCO CONCEPTUAL PARA EL DESARROLLO ECONÓMICO REGIONAL BASADO EN CONOCIMIENTO}

\begin{tabular}{|l|l|}
\hline Etapa de desarrollo & Características \\
\hline Creación de un espacio de & $\begin{array}{l}\text { Se centra en los "entornos de } \\
\text { innovación regionales" donde } \\
\text { diferentes actores trabajan para } \\
\text { mejorar las condiciones locales para la } \\
\text { innovación mediante la concentración } \\
\text { de actividades de I+D relacionadas y } \\
\text { otras operaciones relevantes. }\end{array}$ \\
\hline Creación de un espacio de & $\begin{array}{l}\text { Se generan ideas y estrategias en } \\
\text { una "triple hélice" de múltiples } \\
\text { relaciones reciprocas entre sectores } \\
\text { institucionales (académico, público, } \\
\text { privado). }\end{array}$ \\
\hline Creación de un espacio de \\
innovación & $\begin{array}{l}\text { Intentos de realizar los objetivos } \\
\text { articulados en la fase anterior; es } \\
\text { central establecer y/o atraer capital } \\
\text { riesgo público y privado (combinación } \\
\text { de capital, conocimiento técnico y } \\
\text { conocimiento empresarial). }\end{array}$ \\
\hline
\end{tabular}

Tomado de Etzkowitz, 2002.
Lo más destacable de estas contribuciones teóricas y analíticas es que abren un espacio amplio de aplicación de los sistemas empíricos de innovación a distintos niveles, pero especialmente al regional, al tiempo que ofrecen interesantes ámbitos para desarrollar programas de intervención en los sistemas de innovación basados en conocimiento, mediante actuaciones susceptibles de evaluación pública a través de indicadores disponibles en bases de datos internacionales para los ámbitos internacional, nacional, regional y provincial.

\section{Algunas aplicaciones}

La TH ha desarrollado una base empírica que abarca múltiples estudios de casos sobre las relaciones entre universidad, industria y gobierno, y las transformaciones que han experimentado, relativos a sectores económicos y a campos cognitivos como biotecnología, aeronáutica, ciencias de la computación e instrumentación (Shinn, 2002). Esta diversa producción ha sido recogida en números monográficos de revistas de ciencias sociales especializadas en ciencia y tecnología, $I+D$, innovación o medición empírica, como Research Policy (2006) y Scientometrics (2003).

Dado que la TH se propone como un modelo susceptible de medida empírica Leydesdorff (2003) propone la construcción de indicadores estadísticos para la medición de las principales variables del modelo, y la invitación para que sean usados en la medición de las economías del conocimiento. En la introducción al número monográfico de Scientometrics dedicado a la TH, Leydesdorff y Meyer (2003) señalan al intercambio económico, la organización intelectual y los condicionantes geográficos como dinámicas que interactúan en la economía basada en el conocimiento como sistema complejo. La diferenciación expande el sistema de innovación mientras que la integración performativa ${ }^{5}$ permite a las organizaciones retener la riqueza del conocimiento.

Las relaciones UIG proporcionan una infraestructura en red para los sistemas de innovación basada en el conocimiento. Las relaciones entre las instituciones pueden medirse como variables, mientras que los flujos de interacciones generan una "entropía probabilística" (Leydesdorff, 2003), que puede usarse como un indicador para medir la in- 
formación mutua entre las tres dinámicas institucionales de la TH. Si la entropía es negativa se espera mayor auto organización. Las dinámicas de auto organización pueden estabilizarse temporalmente en el revestimiento de comunicaciones entre agencias implicadas. Se puede distinguir diversas dinámicas de relaciones TH a nivel global, nacional y regional en distintas bases de datos, aplicando el indicador a datos cienciométricos y webométricos, lo que lo dota de gran utilidad para su aplicación a diferentes regiones y sectores, como así se ha hecho.

En efecto, el modelo de la TH se ha usado en el análisis regional comparativo entre Suecia y Holanda (Danell y Persson, 2003), de forma aplicable a otras regiones de las que se dispone de datos. En este caso, los resultados muestran flujos hacia las regiones más industrializadas -que acaparan el $73 \%$ de las actividades y resultados de $\mathrm{I}+\mathrm{D}-$, mientras que las regiones más pequeñas se convierten en exportadoras netas de cerebros y conocimientos hacia las más grandes. Este resultado es, en principio, fácilmente contrastable con otras distribuciones regionales de resultados y recursos de $I+D+i$, por ejemplo en España y Portugal. Otro caso de análisis regional comparativo, también sobre Suecia es Etzkowitz y Klofsten (2005).

Los indicadores "híbridos" (Meyer, Siniläinen y Tinn, 2003) son propuestos para captar la actividad empresarial y colaborativa de los investigadores universitarios. Como la TH predice la emergencia de la universidad empresarial y el entrecruzamiento creciente de actividades entre universidades, industrias y gobiernos, los indicadores hibridos combinan medidas de patentes con datos de encuesta. El indicador de inventiva tecnológica de la investigación que incluye organizaciones de financiación, usuarios y actividades empresariales realizadas por académicos, sirve como modelo simplificado del proceso de innovación. La experiencia del survey de inventores de Finlandia, mostró que las investigaciones académicas más patentadas proceden de investigación financiada públicamente, y, además, que tiende a ser más utilizada por las grandes firmas más que por las empresas start-up fundadas por empresarios académicos.

En la medida en que las universidades comienzan a ser consideradas (y medidas) como promotoras del desarrollo socioeconómico, la TH proporciona aplicaciones importan- tes en el campo del desarrollo regional, especialmente en el contexto de las regiones periféricas que actualmente no están reconocidas como tecnológicamente dinámicas. En estas áreas, el sistema productivo se basa ampliamente en las PYME de funcionamiento tradicional, en combinación con escasez de inversión en I+D y debilidad del sistema de apoyo institucional. El modelo evolutivo de triple hélice sugiere que en estas regiones existen posibilidades de desarrollo basado en el conocimiento, destacando el papel que pueden desempeñar las universidades como factor de desarrollo económico dentro de una interacción espiral trilateral entre universidad, industria y gobierno (Fondazione Rosselli, 1995; Etzkowtiz, 2002c; Martin y Etzkowitz, 2000; Gebhardt y Etzkowitz, 1996).

Como instrumento para las políticas públicas de innovación basada en conocimiento, la TH se adapta a medidas institucionalistas, por ejemplo Bennera y Sandstörm (2000), que analizan cómo los cambios en el sistema de normas institucionales de financiación traen consigo cambios en el énfasis sobre el potencial comercial y la relevancia para la sociedad de la investigación financiada, así como los efectos sobre las condiciones de trabajo de los investigadores que resultan como resultados de los procesos de negociación sobre la regulación normativa de la investigación académica.

Por último y brevemente, mencionar que desde España se han realizado algunas aportaciones a la amplia red internacional de la TH. Por ejemplo, desde la red de ciencia, tecnología y sociedad del CSIC, Molas, Castro y Fernández (2007) hacen un interesante análisis de las estructuras de interfaz desarrolladas en España y el Reino Unido, señalando a las diferencias culturales que dificultan la comunicación e interacción entre los ámbitos institucionales de la triple hélice. Otro ejemplo es la aplicación del modelo de la TH al análisis del sistema de innovación de Cataluña (Piqué et al., 2006).

\section{Balance crítico: fortalezAs y puntos dÉBILES}

El enfoque de la Triple Hélice no deja de ser una corriente analítica más en la tendencia que desde finales del siglo XX viene preconizando cambios importantes en las funciones y papeles sociales de los conocimientos 
científico-tecnológicos y de sus principales fábricas o factorias: las universidades. Por ello, este enfoque implica atender conjuntamente a sus tres palas - universidad, industria y gobierno- y analizar sus interacciones, así como atender a las organizaciones hibridas a las que han dado lugar.

Hay un cierto consenso en las ciencias sociales relativo a que las sociedades más ricas del planeta desarrollan cada vez más intensamente industrias basadas en el conocimiento y tienden a ser sociedades del conocimiento, concepto a su vez sometido a diversas definiciones y críticas (véase González, 2002). La ciencia industrial y las industrias científicas buscan suministrar a los mercados productos que generen beneficios cuanto más grandes, mejor, al igual que cualquier otro proceso industrial orientado al mercado. El enfoque de la TH ofrece una metodología o herramienta para que, desde el sector público, se emprendan acciones encaminadas a potenciar la economía del conocimiento en un territorio especifico.

Sin embargo, la TH no sólo se concibe como un instrumento o metodología de intervención pública, sino también pretende ser una teoría sociológica sobre las causas y efectos de las innovaciones, especialmente las basadas en conocimiento. Prueba de ello ha sido el constante desarrollo de la perspectiva tanto en el ámbito teórico como empírico. Las diferentes aportaciones internacionales a la red $\mathrm{TH}$ a lo largo de la última década permiten afirmar el gran impacto que ha tenido el enfoque TH tanto en la literatura académica como en la de consumo de gestores e interventores públicos académicamente asesorados. Si se atiende a las primeras críticas suscitadas por la TH a principios de este siglo, puede constatarse que, en su gran mayoria, han sido recogidas y subsanadas desde la propia perspectiva de la TH. Por ejemplo, para Shinn (2002) la $\mathrm{TH}$ no sobresale en el Social Science Citation Index ni en Internet ${ }^{6}$, aunque reconoce el eco y el impacto de las reuniones internacionales de la $\mathrm{TH}$ en los países en vías de desarrollo.

Pero, además, Shinn considera que las propuestas teóricas de la $\mathrm{TH}$ son, en general, oscuras, que su lenguaje es incomprensible y que estos defectos dificultan que la valoración del modelo. Siete años después, estas objeciones han perdido vigencia, pues los trabajos de Etzkowitz, Leydesdorff y otros autores desde 2003 en adelante se han esforzado por aclarar conceptos, hipótesis y mecanismos de medida empírica que permitan controlar las afirmaciones teóricas. Shinn también considera problemática la adecuación de la teoría con los datos empíricos, dado que la teoría de la co-evolución describe estructuras y transformaciones sobre un metanivel y en términos macroscópicos, por lo que las unidades de análisis mantienen un alto grado de agregación, generalización y abstracción (Shinn, 2002: 606-7), el cual impide que el marco analítico encaje con los estudios empíricos inspirados por la TH. Ello hace necesario el desarrollo de mecanismos de intermediación que vinculen los cambios cognoscitivos, económicos e institucionales con la teoría coevolutiva. También respecto a este punto puede decirse que el enfoque de la TH ha avanzado notablemente, especialmente en las diversas aportaciones realizadas en los números monográficos de Research Policy (2006) o Scientometrics (2003).

Por otra parte, Shinn (2002) también señala el carácter contradictorio de algunas afirmaciones sostenidas por los autores de la TH. Por una parte, se dice que la TH se centra en la universidad tradicional a la que se añaden nuevas funciones, discursos y acciones (Etzkowitz y Leydesdorff, 2000), mientras que en la III Conferencia internacional de la TH de Río de Janeiro (2001), Etzkowitz señaló que la TH se encarna en las "incubadoras" o en la "segunda revolución académica". Por otra parte, cuando se postula la "transición interminable" como una serie de pequeños cambios y coevoluciones que tienen lugar dentro de una de las palas de la hélice, parece oponerse al carácter rupturista y único de la TH como una emergencia novedosa, una neo-diferenciación radical. Sin embargo, no hay nada contradictorio en ambas consideraciones de la $\mathrm{TH}$, dado que en algunos casos la TH se presenta como un discurso descriptivo que lleva implícito un mensaje ejemplificador de "buenas prácticas", y en otros como un discurso prescriptivo y performativo encaminado al logro de objetivos deseables. De las implicaciones teóricas y heurísticas de este doble plano discursivo son conscientes los creadores del enfoque TH (por ejemplo, Leydesdorff, 2001; Leydesdorff y Meyer, 2003 y 2006).

Otro crítico de la primera mitad de la década, Elzinga (2004), sitúa a la TH dentro de las corrientes que tratan del "nuevo contrato social" de la ciencia, es decir, de los cambios de finales del siglo pasado en las políticas de la 
ciencia, desde el fin de la guerra fría en adelante (1989). Define la nueva situación de la investigación científica mediante los siguientes rasgos: internacionalización y globalización; financiación externa e investigación propietaria; investigación estratégica y esfuerzos de previsión; vinculación de la ciencia con los intereses comerciales y normas en conflicto entre lo académico y lo comercial. En este nuevo panorama, la TH comparte con otros modelos, como el Modo 2 (Gibbons et al., 1994), la ciencia posnormal (Ravetz, 1999) o la ciencia posacadémica (Ziman, 1994), las siguientes deficiencias:

1) selecciona sólo una pequeña parte del panorama total de la investigación;

2) estiliza las condiciones y los sucesos reales para producir una imagen de un espacio de agencia abstracto (intersticial);

3) exagera la comunalidad y el consenso entre los distintos actores y partes implicadas, al tiempo que atenúa la diferenciación y el conflicto de intereses que surge de la especificidad sistémica especifica;

4) introduce un déficit reflexivo en el que el modelo interactivo que sustituye al modelo lineal fracasa para tomar en cuenta el contexto y la fuerza de la globalización y su impacto sobre las condiciones de investigación;

5) pierde la dimensión epistémica agrupando ciencia y tecnología en una noción reduccionista de "tecnociencia";

6) sustituye la política de la ciencia por política de innovación (Elzinga, 2004: 290).

Los puntos 3 y 4 de la crítica de Elzinga merecen consideración especial, pues se refieren a aspectos que la TH considera como distintivos y necesarios para dar cuenta de los procesos de innovación. En lo que respecta al excesivo énfasis en la comunidad y el consenso, y el correspondiente debilitamiento de la atención en la diferenciación y los conflictos de intereses, puede decirse que nada impide desde la TH el análisis de relaciones conflictivas. Es más, el propio modelo las fomenta en la medida en que las triples hélices, a diferencia de las dobles hélices, son inestables, pueden tener consecuencias no esperadas ni deseadas, y son cambiantes (Leydesdorff, 2001). La TH es un modelo dinámico que obliga a atender constantemente a la densidad de las interacciones y a sus consecuencias en cada una de las palas de la hélice.
En segundo lugar, respecto al punto 4 que señala la imposibilidad del modelo interactivo para tomar en cuenta los límites impuestos por los aspectos macro, como los efectos de la globalización sobre las condiciones de la investigación, cabe decir que el modelo sistémico no impide definir en las condiciones del entorno parámetros sensibles a estos efectos. Un ejemplo podría ser atender a la precarización de las condiciones de trabajo de investigación, el despojo de los derechos de propiedad intelectual que es resultado del trabajo intelectual de investigadores e investigadoras (Fuller, 2001), que ya fueron denominados por Weber (1919) como "proletarización" de los científicos, para referirse a la situación o estatus adecuado a las condiciones capitalistas en que se empezaba a desarrollar la ciencia industrial y una incipiente industria de la ciencia que será clave a finales de ese siglo, como puede verse con facilidad.

Por último, debe señalarse que las críticas de Shinn no hacían sino repetir y reelaborar los clásicos argumentos de crítica a los estudios micro y al enfoque interaccionista que tanto se usaron en los años ochenta del pasado siglo con las controversias entre micro y macro, acción y estructura, y otras dualidades caras a los teóricos sociales (González, 1991).

Para finalizar este balance de críticas a la $\mathrm{TH}$, hay que mencionar a otro crítico relevante, John Ziman (1996), quien destaca algunos rasgos de la ciencia posacadémica en los que encaja la $\mathrm{TH}$, especialmente los que se refieren a los cambios en el entorno en el que se producen las investigaciones, que han supuesto cambios radicales en muchas prácticas y actitudes tradicionales. En primer lugar, el logro individual se está sustituyendo por la acción colectiva de equipos multidisciplinares. Además, la comunicación se ha acelerado telemáticamente y llega a ser instantáneamente global. La sofisticada instrumentación de que se dispone hoy, por último, hace mucho más fácil y muchísimo más caro hacer buena ciencia. Es una metamorfosis completa de la cultura de la ciencia y es debida, dice Ziman, a que los gobiernos, que son los que financian una parte importante de la investigación académica, están poniendo controles cada vez más estrictos para la financiación y quieren conseguir más a cambio. Entre las nuevas exigencias están: mayor transparencia, más responsividad ante las necesidades sociales y mayor implicación en la calidad y el impacto de sus productos. 
Estos nuevos requerimientos son bastante ajenos y extraños al ethos de la ciencia (Merton, 1942) y la están transformando culturalmente.

Dado que la TH se propone precisamente como un modelo para esta nueva situación, como metáfora de un desiderátum a medida de los fines gubernamentales y de las agencias de financiación, hay que valorarla como tal. $Y$, en este sentido, hay que señalar su idoneidad y su oportunidad para adecuarse a distintas regiones del globo, con condiciones particulares y complejas.

Sin embargo, como teoría sociológica de la innovación, como marco analítico para atender a procesos de cambio social relacionados con cambios tecnológicos, habria que decir que el enfoque de la TH es demasiado joven, apenas una década de vida, y aún no se dispone de suficiente material empírico para su contrastación. Sin embargo, hay escasos aunque valiosos intentos de someter a prueba su adecuación e impacto en ámbitos distintos?.

Por otra parte, la TH ha mostrado su afinidad para contribuir a la solución de los problemas actuales en los ámbitos medioambiental y social (Etzkowitz y Zhou, 2006). Por un lado, exportando el modelo hacia la creación de otras triples hélices que atiendan a problemas sociales acuciantes en distintas partes del planeta. Por otro, poniendo a trabajar a las entidades TH en soluciones científicas y tecnológicas (lo que incluye ciencias y tecnologías sociales -como soluciones organizativas o entornos normativos-) a problemas demandados por una organización, la sociedad civil o las administraciones públicas a sus múltiples escalas (de lo local a lo internacional).
En este sentido, puede esperarse gran proliferación de trabajos dentro del enfoque TH en la VII Conferencia Internacional de Glasgow de este año 2009. Será entonces la ocasión de valorar sus aportaciones teóricas, la fortaleza de sus herramientas de medida, la resistencia de sus políticas en momentos de crisis económica y recesión mundial como los actuales, y su eficacia como instrumento real de desarrollo regional sostenible basado en conocimiento.

Por último, hay que plantear una cuestión importante a la que no se ha prestado la atención debida en este trabajo. Es un tema que requiere un análisis específico, pero cuya relación con las hipótesis y premisas de la TH obliga al menos a dejar constancia de su existencia. Se trata de la respuesta de la universidad, de las universidades, al reto de la TH. La gran mayoría de universidades, especialmente las más antiguas, desarrollan sus actividades en un ambiente bastante diferente al de las universidades empresariales a las que se refiere preferentemente el modelo de la TH. Sin embargo, al menos en el ámbito de la Unión Europea, las transformaciones que se están realizando en el marco del Espacio Europeo de Educación Superior (EEES) fruto de los acuerdos de Bolonia y los compromisos de Lisboa, sólo se podrán empezar a evaluar a partir de la próxima década. Pero las diferencias culturales entre las universidades tradicionales y las universidades empresariales se han puesto de manifiesto en algunas ocasiones (Tuunainen, 2005; González y González, 2005), lo que señala a un ámbito empírico al que se deberá prestar atención en el futuro próximo.

A falta de nuevas aportaciones y análisis, se podría acabar este trabajo dejando en el aire la pregunta de si la TH no será otro "viejo vino en nuevas botellas" Weingart (1997).

\section{NOTAS}

1 DARPA son las iniciales en inglés del proyecto que dio lugar a la comunicación entre ordenadores, origen de lo que después pasó a ser Internet y la red de redes.

2 No se considerarán en este trabajo las aportaciones de Leydesdorff a la cienciometría, que merecen análisis propio, aunque se hará alguna re- ferencia en el apartado 3 más adelante.

3 Es decir, las asunciones contenidas en Parsons (1966) y analizadas en Savage (1981) acerca de la teleología (y una cierta teología) presente en el marco parsoniano que impone un sentido al esquema evolutivo de los sistemas sociales, señalando una dirección del cambio proporcionada por la jerarquía cibernética de con-
Aceptado: 15 de marzo de 2009

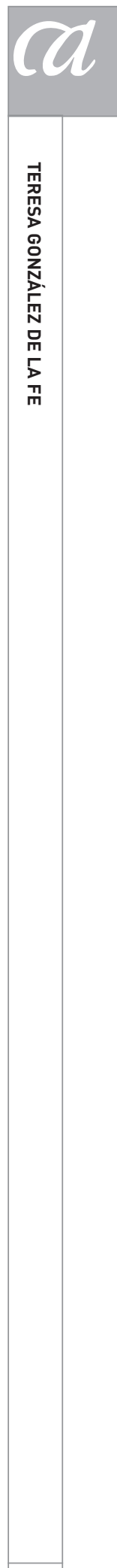


trol (por la cual los sistemas ricos en información controlan a los sistemas ricos en energía), de tal modo que los subsistemas culturales son los que marcan las pautas del resto de subsistemas: sociales, de personalidad y orgánico. Cfr. González (2002), pp. 273 y ss.

4 AGIL y LIGA son los conocidos acrónimos de los cuatro prerrequisitos funcionales de Adaptation, Goals, Integration y Latency de Parsons (1951) y la evolución de la jerarquía cibernética de control.

5 Con "performativa" se hace referencia a las acciones que consisten en lenguaje o formulaciones verbales, tales como las promesas o los compromisos, además de los clásicos actos de habla como los bautizos de personas y buques.

6 En la actualidad usando el motor de Google, la serie triple + helix + etzkowitz + leydesdorff obtiene 7.940 entradas, mientras que triple + helix + leydesdorff obtiene casi 11.000, triple + helix + etzkowitz obtiene 12.200, triple + helix + innovation obtiene aproximadamente 54.600 y triple + helix + university + industry + government + relations obtiene aproximadamente 155.000 .

7 Por ejemplo, Tuunainen (2005) sobre la vida de una empresa híbrida en una universidad tradicional, Garret-Jones y Turpin (2007) sobre las cooperativas de centros de investigación australianas, Molas, Castro y Fernández (2007) para las redes OTRI (Oficinas de Transferencia de Resultados de Investigación dependientes de las universidades y centros de I+D en España) y DDA (Defence Diversification Agence, creadas en el Reino Unido para la mejorar las relaciones del Gobierno en defensa de los centros de investigación y su entorno socioeconómico).

\section{REFERENCIAS BIBLIOGRÁFICAS}

Albert, M. y Laberge, S. (2007): "The Legitimation and Dissemination Processes of the Innovation System Approach: The Case of the Canadian and Québec Science and Technology Policy", Science, Technology and Human Values, n. 32 , pp. 221-249.

Bennera, M. y Sandström, U. (2000): "Institutionalizing the triple helix: research funding and norms in the academic system", Research Policy, vol. 29-2, pp. 291-301.

Braczyk, H.-J.; Cooke, P. y Heidenreich, M. (eds.) (1998): Regional Innovation Systems, London, UCL Press.

Carlsson, B. (1997): "On and Off the Beaten Path: The Evolution of Four Technological Systems in Sweden", International Journal of Industrial Organization, vol. 15, pp. 775-99.

Carlsson, B. y Stankiewicz, R. (1991): "On the Nature, Function and Composition of Technological Systems", Journal of EvoIutionary Economics, n. ${ }^{\circ}$, pp. 93-118.

Cooke, P.; Uranga, M. G. y Etxebarria, G. (1997): "Regional Innovation Systems: Institutional and Organisational Dimensions", Research Policy, vol. 26, n. ${ }^{\text {s }} 4-5$, pp. 475-91.

Danell, R. y Persson, 0. (2003): "Regional $R \& D$ activities and interactions in the Swedish Triple Helix", Scientometrics, vol. 58, 2, pp. 203-218.

Elzinga, A. (2004): "The new production of reductionism in models relating to research policy", en Grandin, Wormbs y Widmalm (eds.), The Science-Industry Nexus, USA, Science History Publications, pp. 277-303.

Etzkowitz, H. (1989): "Entrepreneurial Science in the Academy: A Case of the Transformation of Norms", en Social Problems, vol. 36, 1, pp. 14-29.

Etzkowitz, H. (1994): "Academic-Industry Relations: A Sociological Paradigm for
Economic Development", en Leydesdorff y Van den Besselaar (eds.).

Etzkowitz, H. (1997): "The Triple Helix: academy-industry-governement relations and the growth of neo-corporatist industrial policy in the U.S.", en S. Campodall'Orto (ed.), Managing Technological Knowledge Transfer, EC Social Sciences COST A3, vol. 4, EC Directorate General, Science, Research and Development, Bruselas.

Etzkowitz, H. (2002a): "Incubation of incubators: Innovation as a triple helix of university-industry-government networks", Science and Public Policy, vol. 29-2, pp. 115-128.

Etzkowitz, H. (2002b): "Networks of Innovation: Science, Technology and Development in the Triple Helix Era", International Journal of Technology Management and Sustainable Development, vol. 1-1, pp. 7-20.

Etzkowitz, H. (2002c): "The Triple Helix of University-Industry-Government. Implications for Policy and Evaluation", Working Paper 2002-11, ISSN 1650-3821, disponible en http://www.sister.nu.

Etzkowitz, H. (2003): "Innovation in innovation: the Triple Helix of university-industry-government relations", Social Science Information, vol, 42, n. ${ }^{\circ}$ 3, pp. 293-337.

Etzkowitz, H. y Carvalho de Mello, J. M. (2004): "The rise of a triple helix culture: Innovation in Brazilian economic and social development", International Journal of Technology Management and Sustainable Development, vols. 2-3, pp. 159-171.

Etzkowitz, H.; Carvalho de Mello, J. M. y Almeida, M. (2005): "Towards 'metainnovation' in Brazil: The evolution of the incubator and the emergence of a Triple Helix", Research Policy, 34, pp. 411-424.

Etzkowitz, H. y Klofsten, M. (2005): "The innovating region: toward a theory of 
knowledge-based regional development", R\&D Management, vol. 35, 3, pp. 243-255.

Etzkowitz, H. y Leydesdorff, L. (2000): "The dynamics of innovation: from National Systems and 'Mode 2' to a Triple Helix of university-industry-government relations", Research Policy, vol. 29, n. 2, pp. 109-123.

Etzkowitz, H. y Zhou, C. (2006): "Triple Helix twins: innovation and sustainability", Science and Public Policy, vol. 33, 1, pp. 77-83.

Fondazione Rosselli-CES\&T (1995): Analysis of the regional science \& technology policies in Europe, (CE-DG XII, Grant Contract: PSS*0819), Fondazione Rosselli Scientific Report, 23.

Fuller, S. (2001): "Republicanism as a Theory of Science Governance", en Science under Pressure, Proceedings, The Danish Institute for Studies in Research and Research Policy. Disponible en http://www.afsk.au.dk/ftp/Science_under_pressure/2001_1.pdf.

Garret-Jones, S. y Turpin, T. (2007): "The Triple Helix and institutional change: Reward, risk and response in Australian Cooperative Research Centres", disponible en http://ro.uow.edu.au/ commpapers/327.

Gebhardt, C. y Etzkowitz, H. (1996): "Regional innovation Organiser: a quasi-public role for transational corporations and universities", en Management and New Technology, COST A3, Madrid.

Gibbons et al. (1994): The New Production of Knowledge: The Dynamics of Science and Research in Contemporary Societies, London, Sage.

González de la Fe, T. (1991): "Los problemas perennes de la sociología", en González de la Fe (ed.), Sociología: Unidad y Diversidad, Madrid, CSIC.

González de la Fe, T. (2002): "Conocimiento, tecnologías de la comunicación y cambio social", en M. Beltrán (comp.),
La sociedad: teoría e investigación empírica, Madrid, CIS.

González de la Fe, T. y Sánchez, J. (1988): "Las sociologías del conocimiento científico", Revista Española de Investigaciones Sociológicas, 43, pp. 75124.

González Ramos, A. y González de la Fe, T. (2005): "Especialización cultural y estructura social de las comunidades científicas", Revista Internacional de Sociología, n. ${ }^{\circ}$ 2, pp. 39-67.

Irawati, D. (2006): "Understanding the Triple Helix Model from the Perspective of the Developing Country: A Demand or a Challenge for the Indonesian Case Study?", en MPRA Paper, n. ${ }^{\circ}$ 5829, disponible en http://mpra.ub.uni-muenchen.de/5829/.

Jacobs, M. (2006): "Utilization of social science knowledge in science policy: Systems of Innovation, Triple Helix and VINNOVA", Social Science Information, vol. 45(3), pp. 431-462.

Kadiman, K. (2006): "The Triple Helix and the Public", Jakarta, disponible en http://www.ristek.go.id/index. php?mod=Arsip_Menteri\&conf $=f \&$ fil $e=11122006102431$ _thetriple.pdf.

Kaghan, W. y Barnett, G. (1997): "The Desktop Model of Innovation", en Etzkowitz y Leydesdorff (eds.), Universities in the Global Economy: A Triple Helix of University-Industry-Government Relations, London, Cassell Academic, pp. 71-81.

Lamo de Espinosa (2002): "La sociedad del conocimiento. El orden del cambio", en M. Beltrán (comp.), La sociedad: teoría e investigación empírica, Madrid, CIS.

Leydesdorff, L. (2000): "The triple helix: an evolutionary model of innovations", Research Policy, vol. 29-2, pp. 243255.

Leydesdorff, L. (2001): "Knowledge-based Innovation Systems and the Model of a Triple Helix of University-IndustryGovernment Relations", disponible en http:www.leydesdorf.net.

Leydesdorff, L. (2003): "The mutual information of university - industry - government relations: An indicator of the Triple Helix dynamics", Scientometrics, vol. 58, 2, pp. 445-467.

Leydesdorff, L. (2005): "The Triple Helix Model and the Study of KnowledgeBased Innovation Systems", International Journal of Contemporary Sociology vol. 42, n. ${ }^{\circ}$, pp. 12-27.

Leydesdorff, L. (2006a): "The KnowledgeBased Economy and the Triple Helix Model", en W. Dolfsma y L. Soete (eds.), Reading the Dynamics of a Knowledge Economy, Cheltenham, Edward Elgar, pp. 42-76.

Leydesdorff, L. (2006b): "'While a Storm is Raging on the Open Sea': Regional Development in a Knowledge-based Economy", The Journal of Technology Transfer, vol. 31(1), pp. 189-203.

Leydesdorff, L. y Van den Besselaar, P. (eds.) (1994): Evolutionary Economics and Chaos Theory: New directions in technology studies, Londres, Pinter.

Leydesdorff, L. y Etzkowitz, H. (1996): "Emergence of a Triple Helix of University-Industry-Government Relations", Science and Public Policy, vol. 23, n. ${ }^{\circ}$ 5, 279-286.

Leydesdorff, L. y Etzkowitz, H. (eds.) (1997): A triple Helix of University-IndustryGovernment relations. The future location of Research, Book of Abstracts, Science Policy Institute, State University of New York.

Leydesdorff, L. y H. Etzkowitz (2001a) "A Triple Helix of University-Industry-Government Relations: 'Mode 2' and the Globalization of 'National' Systems of Innovation", en Sience under Pressure, Proceedings, The Danish Institute for Studies in Research and Research Policy. Disponible en http://www. 
afsk.au.dk/ftp/Science_under_pressure/2001_1.pdf.

Leydesdorff, L. y Etzkowitz, H. (2001b): "The Transformation of University-industry-government Relations", Electronic Journal of Sociology, vol. 5, n. ${ }^{0} 4$. Disponible en http://www.sociology. org/content/vol005.004/th.html.

Leydesdorff, L. et al. (2006): "Measuring the knowledge base of an economy in terms of triple-helix relations among 'technology, organization, and territory'", Research Policy, vol. 35, 2, pp. 181-199.

Leydesdorff, L. y Fritsch, M. (2006): "Measuring the knowledge base of regional innovation systems in Germany in terms of a Triple Helix dynamics", Research Policy, vol. 35, 10, pp. 15381553.

Leydesdorff, L. y Meyer, M. (2003): "The Triple Helix of university-industry-government relations", Scientometrics, vol. 58, 2, pp. 191-203.

Leydesdorff, L. y Meyer, M. (2006): "Triple Helix indicators of knowledge-based innovation systems: Introduction to the special issue", Research Policy, vol. 35, 10, 2006, pp. 1441-1449.

Lundvall, B. A. (1988): "Innovation as an Interactive Process: From User-Producer Interaction to the National System of Innovation", en G. Dosi et al. (eds.): Technology and Economic Theory, London, Pinter, pp. 349-69.

Lundvall, B. A. (ed.) (1992): National Systems of Innovation: Towards a Theory of Innovation and Interactive Learning, London, Pinter.

Lundvall, B. A.; Johnson, B.; Andersen, E. y Dalum, B. (2002): "National systems of production, innovation and competence building", Research Policy, vol. 31, 2, pp. 213-231.

Malerba, F. (2002): "Sectoral Systems of Innovation and Production", Research Policy, 31, 2, pp. 247-64.
Martin, B. y Etzkowitz, H. (2000): "The origin and evolution of the university species", VEST, vol. 13, n.os 3-4.

Merton, R. K. (1938/1970): Ciencia, Tecnología y Sociedad en la Inglaterra del siglo XVII, Madrid, Alianza Editorial, 1983.

Merton, R. K. (1942): The normative structure of science, en R. K. Merton, The Sociology of Science: Theoretical and Empirical Investigations, Chicago, University of Chicago Press, 1973.

Meyer, M.; Siniläinen, T. y Utecht, J. T. (2003): "Towards hybrid Triple Helix indicators: A study of university-related patents and a survey of academic inventors", Scientometrics, vol. 58, 2, pp. 321-350.

Molas, J.; Castro, E. y Fernández, I. (2007): '"Interface Structures': a response to the challenges of promoting 'Triple Helix'", disponible en http://digital. csic.es/bitstream/10261/10169/1/ AC165_1_Jordi-Elena-Ignaciomayo-2007-Singapur.presentation6thTripleHelix\%2520paper.pdf.

Nowotny, H. et al. (2001): Re-Thinking Science: Knowledge and the Public in an Age of Uncertainty, London, Polity Press \& Blackwell Publishers.

OCDE (1996): OEDC Economic Outlook, n. ${ }^{\circ}$ 60, París, OCDE.

Parsons, T. (1951): El sistema social, Madrid, Alianza, 1966.

Parsons, T. (1966): Sociedades. Perspectivas evolucionistas y comparativas, México, Trillas, 1974.

Piqué, J. M.; González, S.; Bellavista, J. y Alves, V. (2006): "Parques científicos y tecnológicos y universidades en el sistema de incubación de empresas de base tecnológica: contribución desde el modelo de la Triple Hélice", Coneixement i Societat: Revista d'Universitats, Recerca i Societat de la Informació, n. 11 , pp. 32-47.
Ravetz, J. (1999): "What is Post Normal Science?", Futures, n. 31 , pp. 647-653. Saad, M. y Zawdie, G. (2005): "From technology transfer to the emergence of a triple helix culture: the experience of Algeria in innovation and technological capability development", Technology Analysis and Strategic Management, vol. 17, 1, pp. 89-103.

Savage, S. (1981): The theories of Talcott Parsons. The social relations of action, Londres, Macmillan Press.

Shinn, T. (2002): "The Triple Helix and New Production of Knowledge: Prepackaged Thinking on Science and Technology", Social Studies of Science, vol. 32, 4, 599-614.

Schumpeter, J. (1954): History of Economic Analysis, editado por E. Boody, New York, Oxford University Press.

Schumpeter, J. (1942): Capitalismo, Socialismo y Democracia. Ediciones Folio, Barcelona, 1996.

Tuunainen, J. (2005): "Contesting a Hybrid Firm at a Traditional University", en Social Studies of Science, vol. 35, 2, pp. 173-210.

Viale, R. y Ghiglione, B. (1998): "The Triple Helix model: a Tool for the Study of European Regional Socio Economic Systems", The IPTS Report, vol. 29, European Commission.

Viale, R. y Campodall'Orto, S. (2002): "An evolutionary Triple Helix to strengthen academy-industry relations: suggestions from European regions", Science and Public Policy, vol. 29, 3, pp. 154-168.

Viale R. y Etzkowitz, H. (2004): "Third Academic Revolution: Polyvalent Knowledge: The 'DNA' of the Triple Helix", en http://wwww.triplehelix5. com/files/thc5_themepaper.pdf.

Weber, M. (1919): "La ciencia como vocación", en El político y el científico, edición a cargo de Raymond Aron, Madrid, Alianza, 1986. 
Weingart, P. (1997): "From 'Finalization' to 'Mode 2': old wine in new bottles?", Social Science Information, vol. 36, 4, pp. 591-613.
Ziman, J. (1994): Prometeus Bond. Science in a Steady State, Cambridge, Cambridge University Press.
Ziman, J. (1996): "'Postacademic Science': Constructing Knowledge with Networks and Norms", Science Studies vol. 9, 1, pp. 67-80.

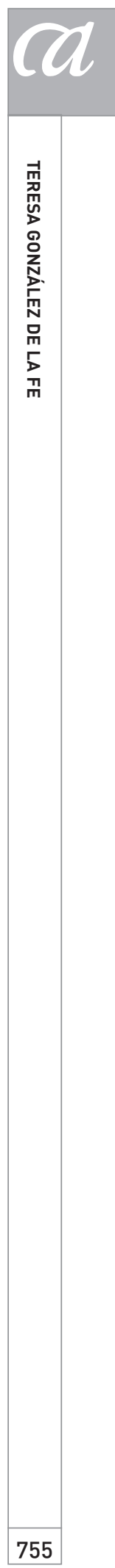

